\title{
Ultrasound of the small joints of the hands and feet: current status
}

\author{
E. G. McNally
}

Received: 8 December 2006 /Revised: 7 May 2007 / Accepted: 12 June 2007 / Published online: 22 August 2007

(C) ISS 2007

\begin{abstract}
The aim of this article was to review the current status of ultrasound imaging of patients with rheumatological disorders of the hands and feet. Ultrasound machines with high-resolution surface probes are readily available in most radiology departments and can be used to address important clinical questions posed by the rheumatologist and sports and rehabilitation physician. There is increasing evidence that ultrasound detects synovitis that is silent to clinical examination. Detection and classification of synovitis and the early detection of bone erosions are important in clinical decision making. Ultrasound has many advantages over other imaging techniques with which it is compared, particularly magnetic resonance. The ability to carry out a rapid assessment of many widely spaced joints, coupled with clinical correlation, the ability to move and stress musculoskeletal structures and the use of ultrasound to guide therapy accurately are principal amongst these. The use of colour flow Doppler studies provides a measure of neovascularisation within the synovial lining of joints and tendons, and within tendons themselves, that is not available with other imaging techniques. Disadvantages compared to MRI include small field of view, poor image presentation, and difficulty in demonstrating cartilage and deep joints in their entirety. Contrast-enhanced magnetic resonance provides a better measure of capillary permeability and extracellular fluid than does ultrasound. The ability to image simultaneously multiple small joints in the hands and feet and their enhancement characteristics cannot be matched with ultrasound, though future developments in
\end{abstract}

E. G. McNally $(\bowtie)$

Department of Radiology,

Nuffield Orthopaedic Centre,

Old Road,

Oxford OX3 7LD, UK

e-mail: eugene.mcnally@ndos.ox.ac.uk
3-D ultrasound may narrow this gap. Magnetic resonance provides a more uniform and reproducible image for longterm follow-up studies.

Keywords Ultrasound · Joint · Rheumatoid arthritis · Synovitis

\section{Introduction}

The small joints of the hands and feet play a central role in the diagnosis and classification of arthropathy. Ultrasound can be used to assess involvement in areas that are clinically occult as well as determine the precise structures involved. Whilst a systematic approach should include a full examination of extra-articular structures, including skin, subcutaneous tissue, the tendon and tendon sheath, the enthesis and associated bursae, this article focuses on intra-articular components of disease, specifically the early detection and classification of effusion, synovitis and erosions. In the patient with arthritis, serial examination can assess current activity and disease distribution, as well as monitoring progression or therapeutic response.

\section{Technique}

The hands are best examined with the patient and examiner seated. The examination table is adjusted to a comfortable height for both, taking account of coexistent shoulder and elbow disease. The examiner sits at $90^{\circ}$ to the patient, giving easy access to both the patient and the ultrasound controls. Good contact and near field resolution require liberal quantities of contact jelly, preferably warmed to body temperature (Fig. 1). Many gel stand-off pads are 
Fig. 1 Position for examination of the hands. The probe is in position for a sagittal examination of the metacarpo-phalangeal joint (MCPJ) on the extensor (a) and flexor (b) sides. The probe is held between thumb and forefinger, with the ulnar border of the hand used as support (arrow). Note the liberal quantity of gel used to provide some stand-off (arrowhead). c Position for examination of radial aspect of second MCPJ. d Probe position to examine the collateral ligaments and lateral compartments of the interphalangeal joints

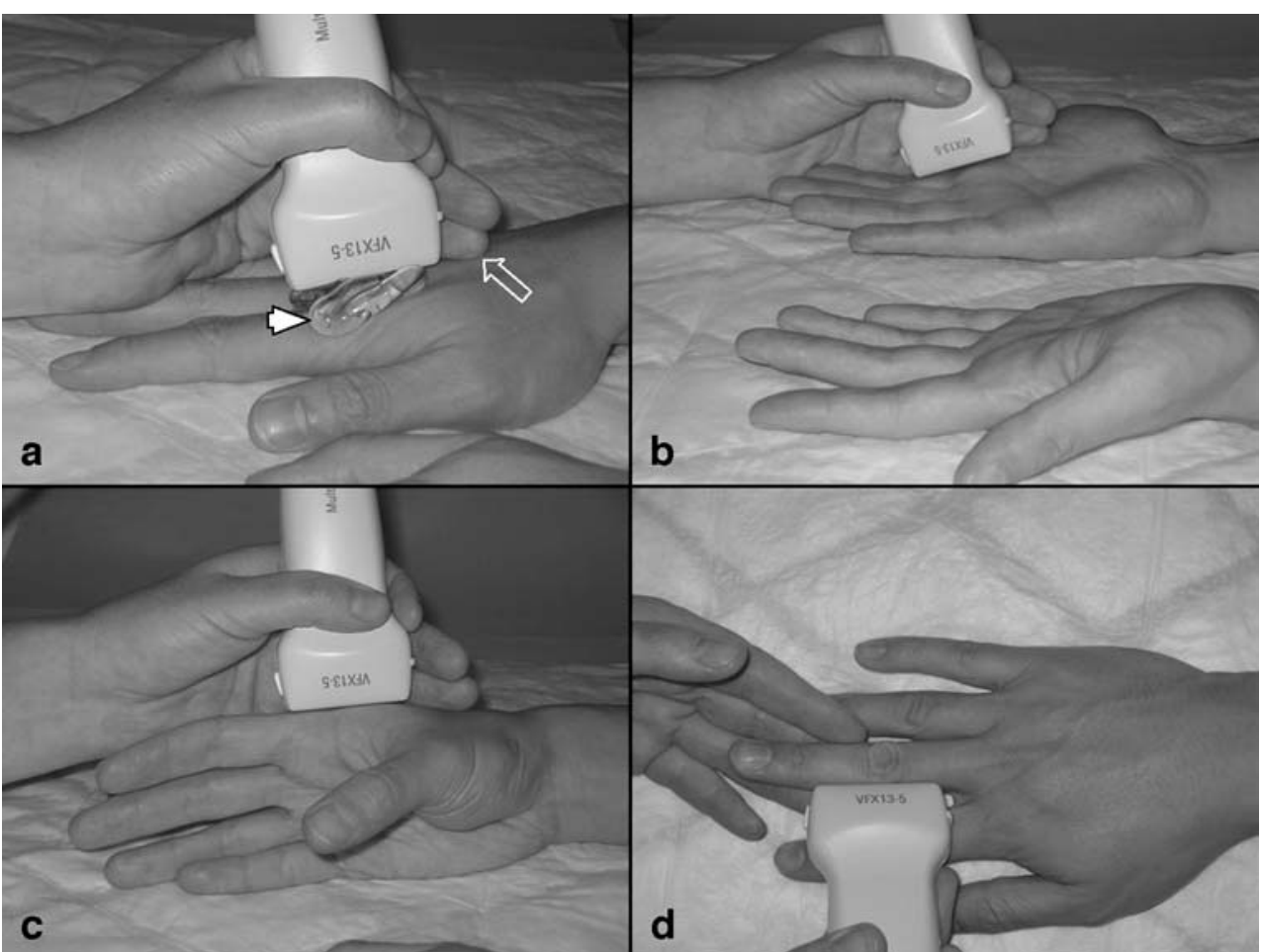

rather awkward and can limit access to the lateral recesses of small joints; soft pads work best. Some operators like to immerse the patient's hand in warm water; if this method is used, it is preferable to leave the water to stand for some while so that all air bubbles are dissipated.

High-frequency linear-array probes are mandatory, probes with operating frequencies of $10 \mathrm{MHz}$ or more providing the best images. The probe should be held lightly between thumb and forefinger (Fig. 1), and the little finger allowed to rest on the table or patient to reduce contact pressure. Excessive probe pressure can obliterate small quantities of fluid, reduce the sensitivity for detection of blood flow and may obscure synovitis. Sagittal images are the mainstay for diagnosis, with axial [metacarpo-phalangeal joint (MCPJ)] and coronal [proximal interphalangeal joint (PIPJ)] images used in support. The superficial structures, including skin, subcutaneous tissue tendon and tendon sheath, should be assessed prior to the joints themselves. Within the joint, the capsule, extra-synovial connective tissue structures, synovium and visible cartilage are examined in turn. The joint should then be moved gently, as it is only during movement that some of the interfaces between the normal structures become sharply defined. Movement may also facilitate detection of low-volume synovial thickening, which bunches up in the proximal extensor recess on flexion.

A comprehensive examination of the small joints of the hands and feet can be time-consuming; however, efficiency can be improved without compromising diagnostic impact, by omitting joints that are commonly negative or misleading. The first ray of both hands and feet often contain effusions, synovial thickening and osteophyte formation in the asymptomatic population. Clinically occult synovial thickening is found more commonly in the PIPJs than in the distal interphalangeal joints (DIPJs) of patients with rheumatoid arthritis (RA) [1]. Unless specifically symptomatic, the DIP joints are not routinely examined in patients with RA, but are included in patients with osteoarthritis or seronegative arthritis or where the diagnosis is unknown, in which cases the tendon insertions should also be included to look for enthesopathy.

For a routine rheumatological examination of the hand, the author examines the second to fifth rays. The first ray is included only in specific clinical situations (Fig. 2). The extensor side of the metacarpo-phalangeal joints are examined first, followed by the extensor, ulnar and, in particular, the radial aspects of the IPJs, as indicated (Fig. 1). The dorsal aspects of the wrist and extensor tendons are studied before turning to the palmar side. The flexor aspect of the metacarpophalangeal and proximal interphalangeal joints are examined, with particular attention paid to those that have been normal on the extensor side.

Other authors debate whether both the flexor and extensor sides need to be examined. Scheel and co-authors found that, in most cases, synovitis was detected in the palmar and proximal sites of the MCP and PIP joints, with only $14 \%$ of affected joints not showing synovitis in these 


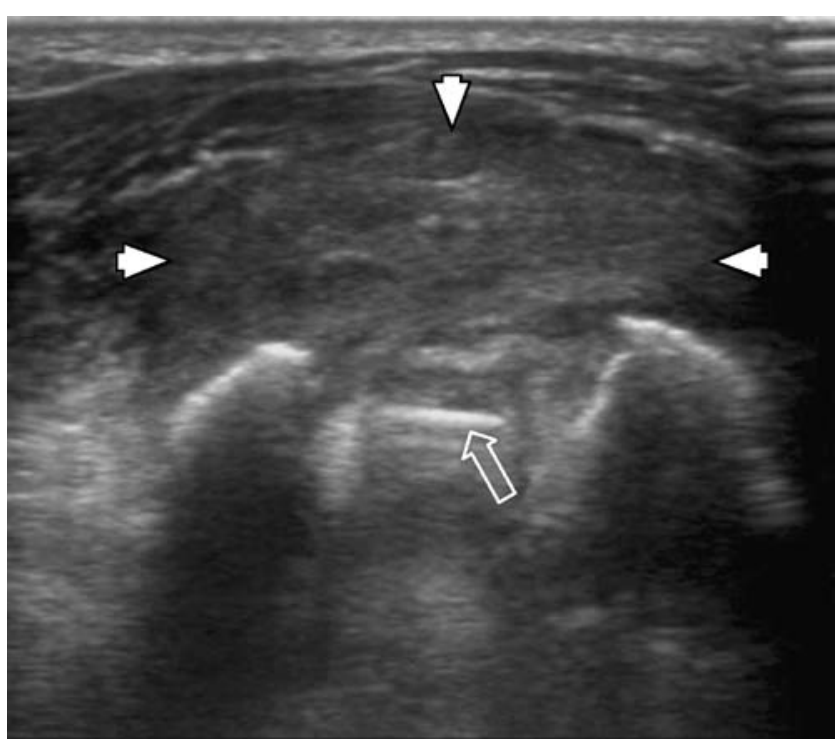

Fig. 2 Iatrogenic synovtis (arrowheads) of the carpo-metacarpal joint due to silastic implant (arrow)

locations [1] Because of this preponderance, they propose that the examination be streamlined to include only the palmar side. Not all authors agree, and other published work suggests that a significant proportion of synovitis would be overlooked if the examination were to be limited to either one or the other. Ostergaard and Szkudlarek found that only a third of patients had synovitis on both palmar and dorsal aspect of the PIPJ. In the majority, synovitis was limited to one or other compartment, with $43 \%$ limited to the palmar [2] and $27 \%$ to the extensor sides. The prevalence is reversed in the metacarpo-phalangeal joints; $80 \%$ of synovial thickening will be detected on the extensor aspect. The distribution of synovitis within MCPJs has also received attention [1-3]. Tan et al. used contrast-enhanced MRI and divided the volume of synovitis surrounding each MCP joint into eight sections. They detected a predilection for radial-sided synovitis in the second and third MCPJs, with equal distribution in both sides in the fourth and fifth MCPJs. The distribution on the dorsal versus palmar sides was not published. Hau and colleagues also found a predilection for radial-sided synovitis in the PIPJs [4]. Scheel et al. tested various combinations of joints to determine the most efficient method of providing an overall synovitis score. Synovitis and effusion were not differentiated. Of the various joint combinations tried, counting the medial four MCPJs only was least sensitive, counting the flexor side of the MCP and PIPJs of fingers 2-4 was most sensitive. Whilst this may work well for an overall synovitis score, omitting the fifth ray may reduce the sensitivity for detecting erosions, as the fifth MCPJ and fifth MTPJ are often involved early.

\section{Normal anatomy}

Metacarpo-phalangeal joints

A sagittal image of the palmar aspect of the metacarpophalangeal joint is shown in Fig. 3. Note the presence of a layer of contact gel between the probe and the underlying skin (Fig. 3b). This provides good resolution of skin and subcutaneous tissues, as well as reducing the amount of probe pressure, which can interfere with the assessment of subtle joint effusion and blood flow. Abnormalities in the superficial layers include increased thickness in psoriatic arthritis and calcification.

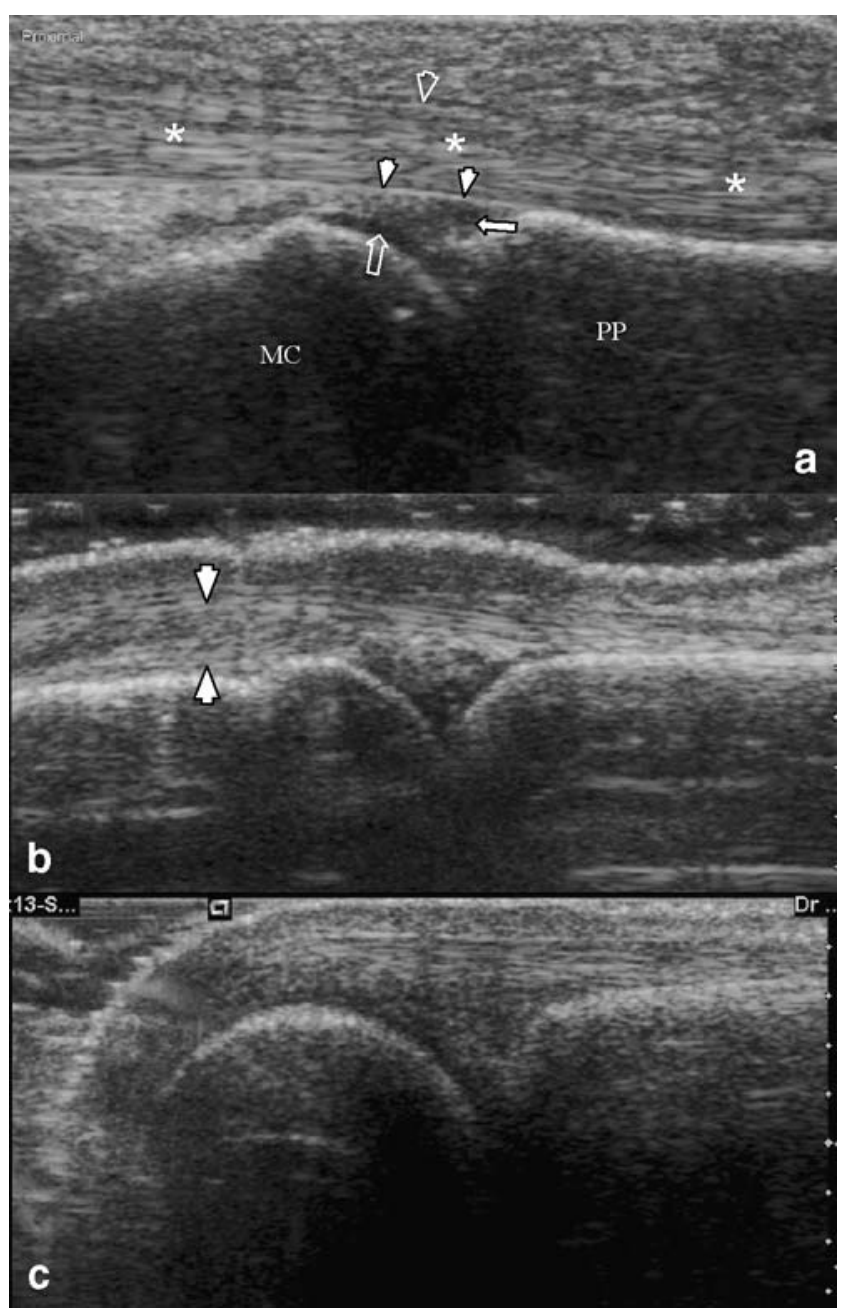

Fig. 3 a Long axis (sagittal) view of the flexor aspect of the metacarpo-phalangeal joint. The flexor tendons (asterisks) are lying on the anterior aspect of the joint capsule (arrowheads) held in place by the cribriform (c) pulley (open arrowhead). The volar plate (arrow) and articular cartilage of the metacarpal head (open arrow) are visible. Extensor aspect in full extension (b) and flexion (c). Flexing the MCPJ compresses the connective tissue in the proximal recess (between arrowheads) and may make subtle synovial thickening more conspicuous. $M C$ metacarpal, $P P$ proximal phalanx 
Deep to this is the flexor tendon in its tendon sheath reinforced by the cruciform pulley. The superficial and deep flexor tendons should be independently identified as they pass over the metacarpo-phalangeal joints into the flexor tendon sheath of the fingers. Differences in tendon excursion on finger movement allows the two tendons to be separated easily. The tendon should pass in close proximity to the proximal phalanges where they are held in position by the annular (A2) pulley. The pulleys can be seen as thin poorly reflective linear structures (Fig. 3). Small fluid collections are often seen in relation to them, which are not usually clinically significant.

The joint capsule on the flexor side is reinforced by several connective tissue structures, which can be identified on ultrasound. The collateral ligament runs obliquely from posterolateral to anterolateral and is best appreciated on coronal images (Fig. 1). The accessory collateral ligament has its origin on the head of the proximal phalanx, between the collateral ligament and volar plate, with an insertion on the volar plate itself (Fig. 4). The volar plate is a centrally positioned fibrocartilagenous structure that has a broadbased attachment to the base of the proximal phalanx (Fig. 3). It inserts by two slips onto the neck of the adjacent metacarpal. These are called the check-rein ligaments. Within the joint, the proximal recess on the flexor aspect of the metacarpo-phalangeal joints is identified. Overlying this is the capsule of the MCP joint, which inserts on the adjacent metacarpal neck some distance from the joint surface. Hyporeflective articular cartilage is identified deep to the volar plate on the metacarpal head.

The proximal recess is the area between the volar aspect of the metacarpal neck and the joint capsule, which abuts the deep surface of the overlying tendon. It is filled with intra-capsular but extra-synovial fat, which normally keeps the two layers of synovium closely approximated to one another. This layer of fat is very much more prominent in the proximal recess on the extensor aspect of the joint, which can extend up to $2 \mathrm{~cm}$. from the joint level. This is to allow for finger flexion [5]. The distal recess is much

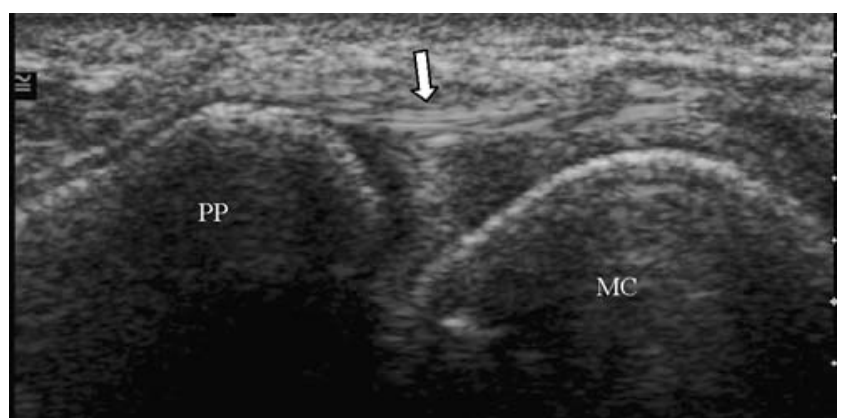

Fig. 4 Coronal image of the second metacarpo-phalangeal joint, showing the radial collateral ligament (arrow). $M C$ metacarpal, $P P$ proximal phalanx smaller, as the extensor tendon conforms to the shape of the proximal phalanx.

Absolute measurements of normal joints have not been universally agreed, largely because different workers have used different joints, different parts of joints and different anatomical structures within the joint to define normal dimensions. The variation in the normal was demonstrated by Scheel and co-authors, who looked at the consensus between ultrasonologists involved in training programmes for the European League against Rheumatism (EULAR) [6]. Many of the differences in interpretation were the consequence of failure to appreciate normal findings. Schmidt et al. proposed a standard measurement, using the distance between the sub-chondral cortex on the head of the metacarpal and the volar plate in the second MCPJ. A mean of $1 \mathrm{~mm}$ was determined in a group of 102 asymptomatic volunteers [7], but variation was found to be quite wide. The extensor compartment was not examined, though this area is commonly screened for synovitis [8]. Furthermore, this measurement does not include the proximal recess, where early and prominent synovial thickening may occur. There are also differences between the proximal and distal recesses and some differences between radial and ulna sides, which cannot be encompassed by this single midline measurement.

For these reasons, others use the point of maximal joint distension and compare it with the normal measurement for that location. This, however, varies with the area of the joint being examined, but most authors agree that an increase in joint dimension of more than $1 \mathrm{~mm}$ above normal is sufficient to suggest abnormality. On the extensor aspect of the MCPJs, the proximal recess can be measured from bone to the deep surface of the extensor tendon, a distance of approximately $2.5 \mathrm{~mm}$. The distal recess is much smaller, measuring less than $1.5 \mathrm{~mm}$. In the transverse plane, the synovial space is limited on its lateral and medial sides, proximally by the extensor hood and distally by the extensor slips. There should be no posterior bulging of the hood or slips; this finding would suggest focal synovial disease. On the flexor side, the proximal recess is more distended and easier to visualise than the distal recess. The proximal recess measures approximately $3 \mathrm{~mm}$ from bone to the deep surface of the tendon. This space includes fat, capsule and two synovial layers, so the space may be increased by hypertrophy of any of these. On high-resolution equipment, the joint cavity itself can be identified between surrounding fat and connective tissue. Occasionally, a small quantity of fluid can be identified, separating the two synovial layers. With increasing practice, ultrasonologists quickly gain an appreciation of the range of normal for the small joints of the hands and feet.

The bony contours of the metacarpal head and proximal phalanx should be smooth, though it is not uncommon to 
identify a normal indentation on the dorsal aspect of the metacarpal head. This depression is smooth, well demarcated and has reflective bone at its base. It occurs at the site of the fused growth plate and is not associated with overlying synovial thickening. These features allow differentiation from a metacarpal head erosion.

Interphalangeal joints

The flexor side anatomy of the proximal interphalangeal joints is similar to that of the metacarpophalangeal joints. The volar capsule is reinforced by similar connective tissue structures. A centrally positioned volar plate has a broadbased attachment to the base of the middle phalanx. It also inserts by two slips onto the neck of the adjacent proximal phalanx. On either side of this lie two collateral ligaments. The accessory collateral ligament has its origin on the head of the proximal phalanx between the collateral ligament and volar plate. The capsule and the flexor tendons lie superficial to these. The medial and lateral extensor tendon slips of the superficial flexor tendon can be followed as they insert just distal to the PIPJ (Fig. 5). The deep flexor tendon can then be followed to its insertion on the base of the distal phalanx. In most cases, it is possible to identify a small quantity of fluid surrounding the tendon, seen as a poorly reflective halo. The thickness of this halo varies from person to person, and comparison with other tendons is helpful, assuming they are uninvolved. Another useful tip is that normal fluid surrounding the tendon is continuous on a longitudinal image, and there is no increase in vascularity, other than at the mesotenon.

Loose adipose tissue, which surrounds the synovial membrane, lies between flexor tendon and bone. Care must be taken not to misdiagnose this fat as synovial thickening, particularly when its reflectivity is artefactually reduced. On high-resolution equipment, the proximal recess on the flexor side can be differentiated from surrounding fat and connective tissue. The sagittal images are augmented by coronal views, which provide the best depiction of the medial and lateral joint recesses and collateral ligaments.

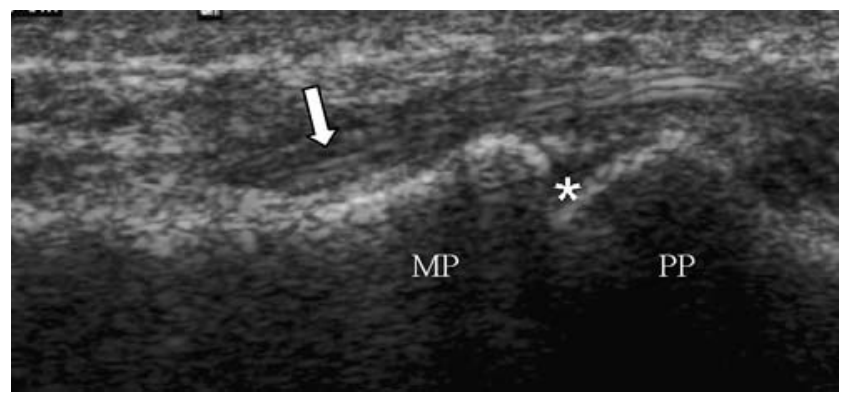

Fig. 5 Long-axis parasagittal view of the proximal interphalangeal joint showing the insertion slips of the superficial flexor tendon (arrow), just distal to the proximal interphalangeal joint (asterisk). PP proximal phalanx, $M P$ middle phalanx
The coronal images are obtained by asking the patient to hyperextend the MCP of the finger being examined (Fig. 1d). Particular attention should be paid to the radial aspect of the joint, where synovial hypertrophy and erosions predominate.

For the PIPJ, measurements can be obtained from the flexor, extensor, radial and ulnar sides. On the extensor side, the distance between the proximal phalanx, at the junction with the head, and the deep surface of the extensor tendon is $1 \mathrm{~mm}$ in the central sagittal plane. On the flexor side the same measurement is $2 \mathrm{~mm}$, with most of the space taken up by intra-capsular but extra-synovial fat. A small quantity of fluid is commonly encountered in this recess (Fig. 6), and slight movement of the joint can make this more conspicuous. Under normal circumstances, the quantity of fluid should not be thicker than the joint capsule and should not extend out of the recess in which it is contained.

Measuring the joint space on the radial and ulnar side is difficult. This is due to the variable appearances of the ulnar and radial collateral ligaments due to anisotropy. For this reason it is often best to measure from the bone surface to the outer aspect of the collateral ligament. Under normal circumstances this distance is less than $2.5 \mathrm{~mm}$.

The wrist joint is often included in rheumatological examination of the hand, although a full description is outside the scope of this review. The joint is best appreciated from its dorsal aspect, where a small amount of fluid is frequently identified in the radiocarpal joint. Because of the undulating surface of the first carpal row there is normally a variation in the bone-to-capsule distance. To provide some standardisation, measurements can be obtained using the deep surface of the extensor tendons or bony structures as landmarks. The radius, lunate and capitate axis are particularly easy to identify (Fig. 7). Normal ranges have not been established by large population studies; however, the author uses a guide measurement of $7 \mathrm{~mm}$. It is emphasised that for larger joints, such as the wrist joint, measurements should only be used as a loose

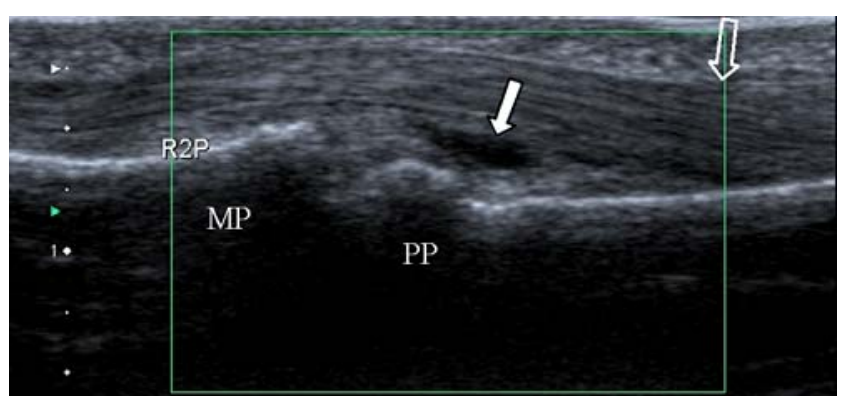

Fig. 6 Long-axis view of the second proximal interphalangeal joint flexor aspect. A small quantity of fluid can be present in the normal joint (arrow). The distal end of the A2 pulley is just visible (open arrow). The fluid is transonic, and the Doppler field shows no flow. $P P$ proximal phalanx, $M P$ middle phalanx, $R 2 P$ annotation on image for right second PIPJ 


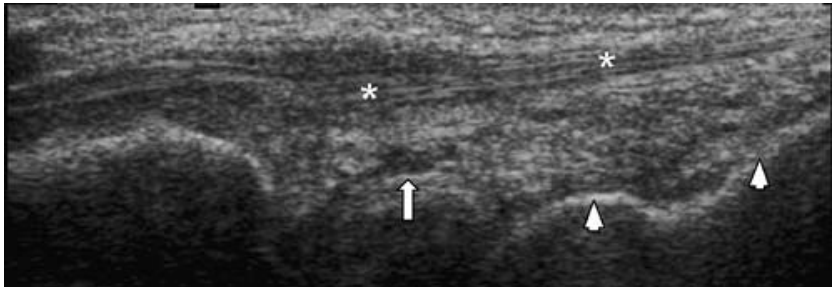

Fig. 7 Sagittal image of the normal wrist joint. Standard sections obtained along the third extensor tendon (asterisks) in the axis of the lunate (arrow) and capitate (arrowheads)

guideline, as normal variation is wide and synovial proliferation may be focal. Once again, comparison with the contralateral side may be of value in unilateral disease.

\section{The small joints of the forefoot}

Technique The metatarso-phalangeal joints and proximal interphalangeal joints of the toes are best examined from the extensor aspect. The big toe is usually not included in the general rheumatological screen, as effusion and bone irregularity are frequently encountered in the asymptomatic population.

Normal anatomy On the dorsal aspect of the metatarsophalangeal joint, extensor digitorum longus and brevis lie superficial to the joint. The extensor digitorum brevis tendon divides into four slips, one to each toe, which run alongside the extensor digitorum longus tendon before inserting into it. The longus tendon itself inserts into the dorsal aspects of the middle and distal phalanges, via the dorsal digital expansions. The dorsal digital expansions have three slips, one central and two collateral. The central slip inserts into the base of the middle phalanx. The collateral slips pass on either side of the inserting central slip and receive reinforcement from the interosseus muscles, the extensor digitorum brevis and the lumbricals. Deep to the extensor tendons, the metatarso-phalangeal joints are reinforced by collateral ligaments that run from dorsal to plantar, crossing the radial and ulnar aspects of the joint. These are difficult to identify individually.

Flexor digitorum longus and brevis tendons lie on the plantar aspect of the forefoot. The flexor digitorum brevis divides into four tendons, one to each of the lateral four toes, and each of these in turn splits into two at the level of the base of the proximal phalanx. The two divisions reunite before dividing again to attach on either side of the middle phalanx. The first division of this tendon is to allow the deeper flexor digitorum longus to pass distally. The tendon sheaths are fibrous tunnels, reinforced by annular and cribriform condensations mimicking the pulleys of the fingers. Running between the metatarso-phalangeal joints are fibrous condensations termed transverse metatarsal bands. Taken as a unit, these are often referred to as the deep transverse metatarsal ligament. Lying directly on the plantar aspect of the joint capsule are the plantar plates (Fig. 8), which are similar to the volar plates of the upper limb. On their deep surface, these blend with the joint capsule. The plantar plates are firmly attached distally to the base of the proximal phalanges. Proximally, there is a rather loose attachment just proximal to the articular cartilage of the head of the metatarsal.

\section{Ultrasound pathology}

\section{Intra-articular pathology}

Effusion and synovitis There is an increasing trend for early and more aggressive treatment of synovitis with diseasemodifying anti-rheumatic drugs (DMARDs), which may demonstrate benefit when prescribed even within weeks to months of the onset of disease [9]. The emphasis in early disease detection has now moved from the detection of erosion to the earlier detection of synovitis, and erosions are now generally regarded as being a stage too late. The earliest detectable abnormality within the small joints of the hands and feet is effusion.

Definitions Simple effusion should be completely transonic, compressible, and with no increase in Doppler signal (Fig. 9). Synovial hypertrophy is defined as nondisplaceable, intra-articular, poorly compressible tissue, which may exhibit Doppler signal (Fig. 10). Dynamic examination using the probe to compress the joint will cause fluid to be displaced away from the probe, whereas thickened synovium will be much less compressible. The most important pitfall is that normal anatomical structures

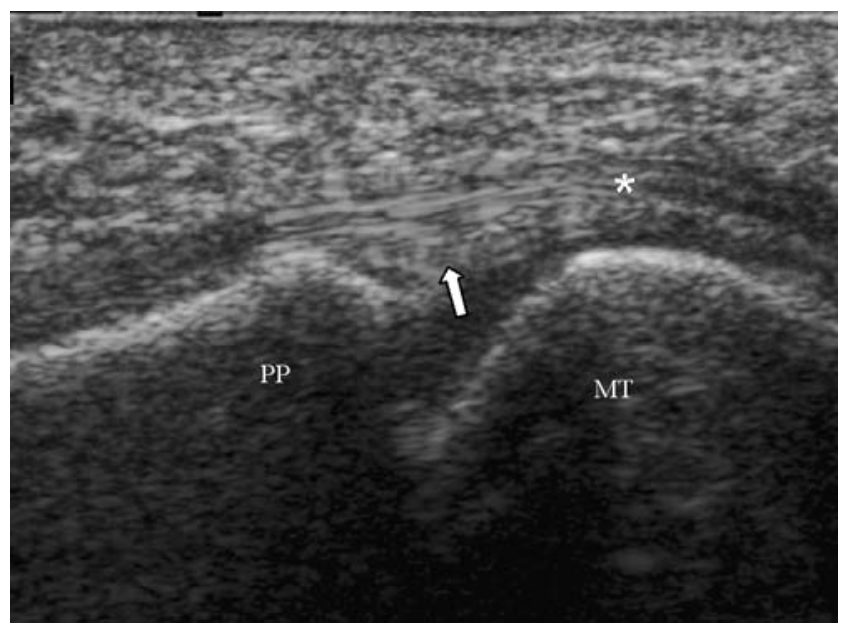

Fig. 8 Long-axis (sagittal) view of the plantar aspect of the metatarso-phalangeal joint. The extensor tendon (asterisk) runs over the plantar plate (arrow). MT metatarsal head, PP proximal phalanx 


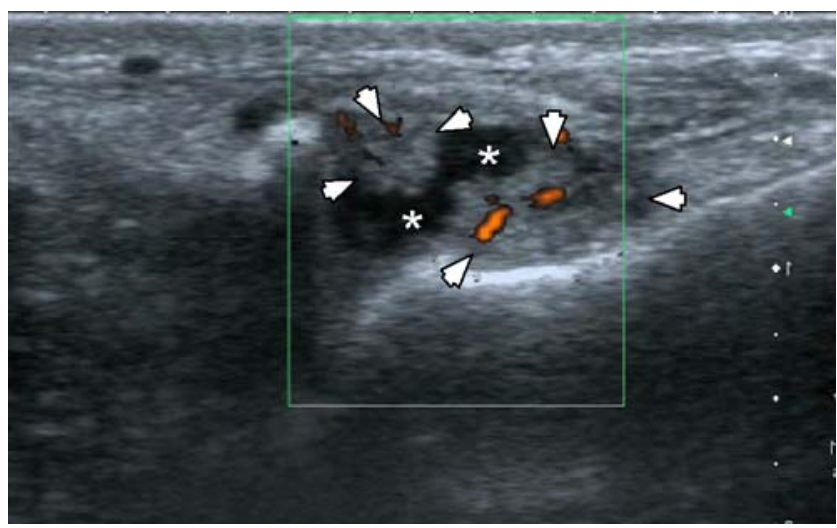

Fig. 9 Small effusion on the flexor aspect of the proximal interphalangeal joint. Fluid thickness is measured at $1.6 \mathrm{~mm}$ and extends out of the proximal recess (arrowheads). Compare with Fig. 6. $P P$ proximal phalanx, $M P$ middle phalanx

may have low reflectivity and mimic synovitis if careful attention is not paid to technique, particularly with lower resolution equipment. In particularly, anisotropy of adjacent capsule ligaments and tendons should be avoided.

Classifying abnormal Synovial hypertrophy has been measured in a number of ways. Szuldarek categorised changes in synovial thickness by comparing it to bony structures. Grade 1 is minimal synovial thickening (considered normal), grade 2, synovial thickening bulging over the line linking the tops of the bones forming the joint without extension along the bone diaphyses, grade 3 , with extension to one of the metadiaphyses and grade 4 , extension to both metadiaphyses.

The author classifies joint disease using semi-quantitative measurements of synovial thickness, vascularity and association with erosions. Synovial thickness is recorded on a 3point scale $(1-3=$ mild, moderate and severe $)$, with moderate

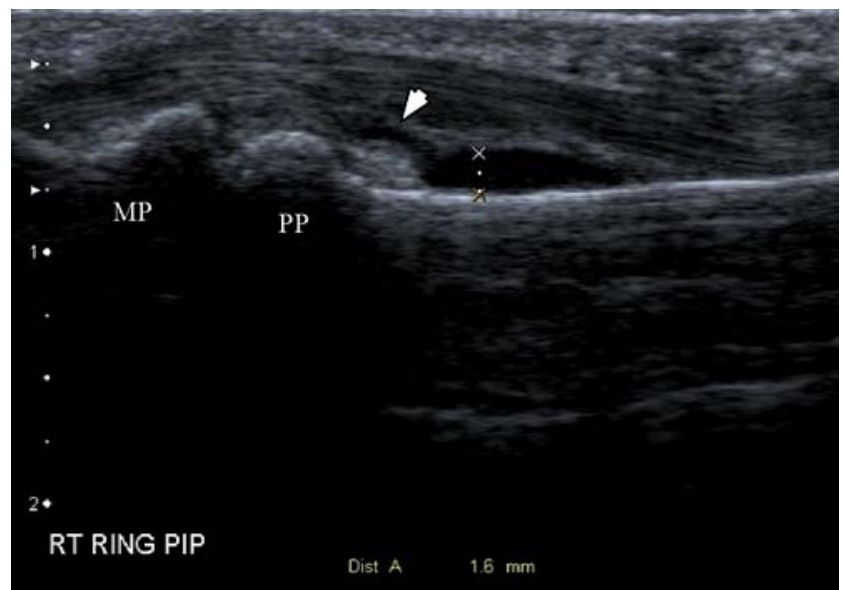

Fig. 10 Moderate synovitis and bloodflow. Compare synovial thickening (arrowheads) with effusion (asterisk). Synovial thickening is hyper-reflective, non-compressible and demonstrates increased vascularity when compared with free fluid synovial thickening between $2 \mathrm{~mm}$ and $4 \mathrm{~mm}$ above normal. If desired, mild grades of synovitis can be further classified into focal and diffuse, with focal involvement limited to one recess. Moderate and severe synovial thickening is less often focal. This sub-classification may be of value in monitoring more subtle changes, compared with the more two-dimensional classification. A 3-point scale is also used to record blood flow: mild is defined as a few scattered vessels only (Fig. 11), moderate as less than $50 \%$ vascularity in the synovium (Fig. 12) and severe as more than 50\% (Fig. 13). Finally, 0 and 1 are used to denote the presence or absence of erosions, though where necessary for record or research purposes, a more detailed description of the proportion of bone involvement by erosions can be used. The score is recorded on the ultrasound image, together with annotation of the joint being measured. In summary, the second right MCPJ, with $3 \mathrm{~mm}$ of synovial thickening, diffusely involving the joint, with marked increased in blood flow and without erosions, would be classified as R3M 230 . Images are printed as hard copy or stored on the department's picture-archiving communication system (PACS) for future comparison and to monitor the patient's progress.

Several studies have shown good inter- and intraobserver reliability of ultrasound classification. The classification by Szkudlarek et al. has been most tested and shown to have good inter-observer agreement. An interobserver correlation coefficient ( ICC) of 0.61 was determined in a review of 150 small joints of the hand in 30 patients [10]. Naredo and co-workers coordinated a study of 22 ultrasonologists and 28 patients. For the detection of effusion and synovitis in the small joints of the hands and wrists, a mean kappa value of 0.61 was calculated [6]. In a study of 204 hands in normal subjects, Schmidt et al. found a reliability of 0.96 in repeat examinations of the same test subject [7]. Ultrasonography (US) classifications have also been shown to correlate reasonably well with findings on MRI in patients with synovitis, but there have been no studies of the variation that may be found in individuals with normal joints.

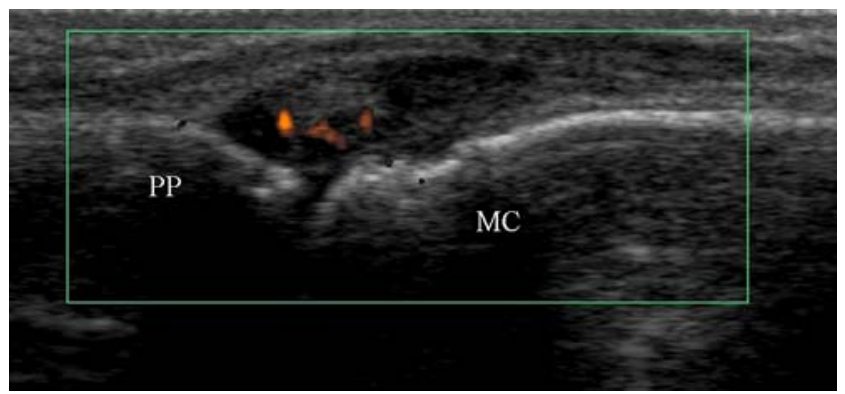

Fig. 11 Sagittal image of the extensor aspect of an inflamed metacarpo-phalangeal joint. Mild hypervascularity with a few scattered vessels visible in the moderately thickened synovium. $M C$ metacarpal head, $P P$ proximal phalanx 


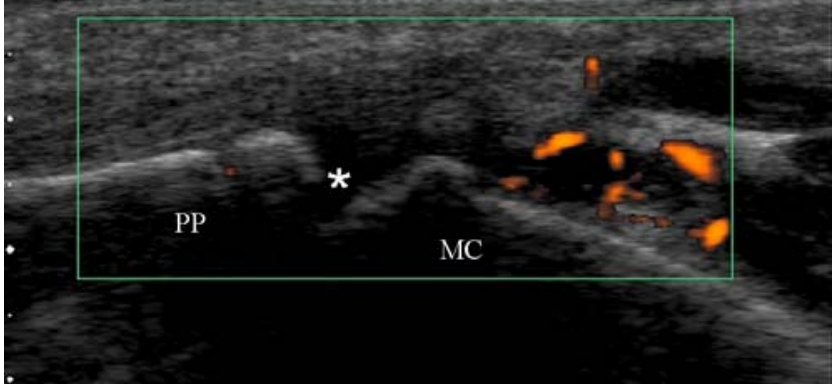

Fig. 12 Sagittal image of the extensor aspect of an inflamed metacarpophalangeal joint (asterisk). Detectable blood flow on power Doppler is seen in less than $50 \%$ of the severely thickened synovium. $M C$ metacarpal head, $P P$ proximal phalanx

\section{Angiogenesis}

High-frequency US can differentiate solid from fluid elements within an enlarged joint space but is less effective at distinguishing inflamed synovium from inactive pannus, fibrous tissue and joint debris. One method of making this differentiation is to use Doppler colour flow, though there has been some variation in the reported findings in individuals with normal joints. Klauser et al. [11] found that healthy joints showed no intra-articular vascularisation, either before or after the administration of ultrasound contrast medium, while Terslev and colleagues [12] found vessels in the synovium of the MCPJs of 11 healthy subjects, in $18 \%$ before and 50\% after administration of contrast agent. Different ultrasound equipment was used, with small differences in probe and Doppler frequencies. Though there is no method of determining for certain whether these differences account for the variation in findings, it is well recognised that sensitivities of ultrasound equipment from different manufacturers vary considerably,

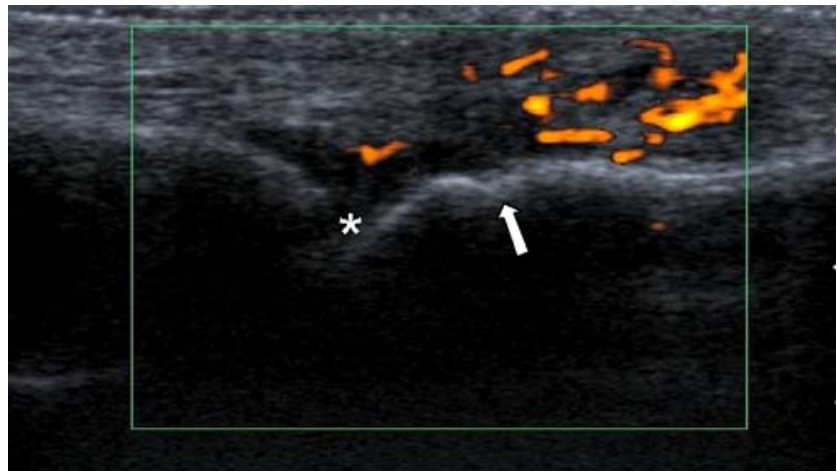

Fig. 13 Sagittal image of the extensor aspect of an inflamed metacarpo-phalangeal joint (asterisk). Detectable blood flow on power Doppler is seen in more than $50 \%$ of the moderately thickened synovium. Note the apparent defect in the metacarpal head (arrow). This is a normal finding and should not be missed or diagnosed as an erosion. Note the well-demarcated floor and lack of through sound transmission and the identification of the occasional blood vessel should probably not be regarded as abnormal when sensitive equipment is being used.

Response to inflammatory changes within the synovium results in the secretion of a large number of inflammatory mediators. Vascular endothelium growth factor (VEGF) is one of the pro-hormones secreted and results in proliferation of the vascular endothelium to form new vessels, a process termed angioneogenesis. Histological studies confirm that power Doppler changes correlate quite well with pathological changes in the synovium [13, 14], although great care should be taken to avoid interface artefact, which can be present at the bone synovium interface if gain settings are too high. Increased Doppler signal correlates especially with polymorphonuclear leucocyte infiltration and surface fibrin deposition [15], though, as yet, no direct correlation between serum VEGF measurement and neovascularisation has been identified.

Colour flow assessment should, therefore, accompany all ultrasound examinations of the swollen joint. The pulse repetition frequency should be kept low, and the region of interest should also be kept as small as possible, to maximise the detection of abnormal blood flow within the synovium. False positive readings can occur, due to patient or operator movement or at highly reflective interfaces, such as between bone and soft tissue (Fig. 14).

There are two ways of measuring synovial blood flow, power Doppler ultrasonography (PDUS) and colour flow Doppler ultrasonography (CFDUS). Power Doppler is more sensitive but also more prone to artefact. It is sensitive to intravenously administered contrast agents which can be used to augment very slow rates of flow. The software that measures power Doppler is proprietary; therefore, results from one manufacturer may not be comparable with another. There is also a concern that serial studies on the

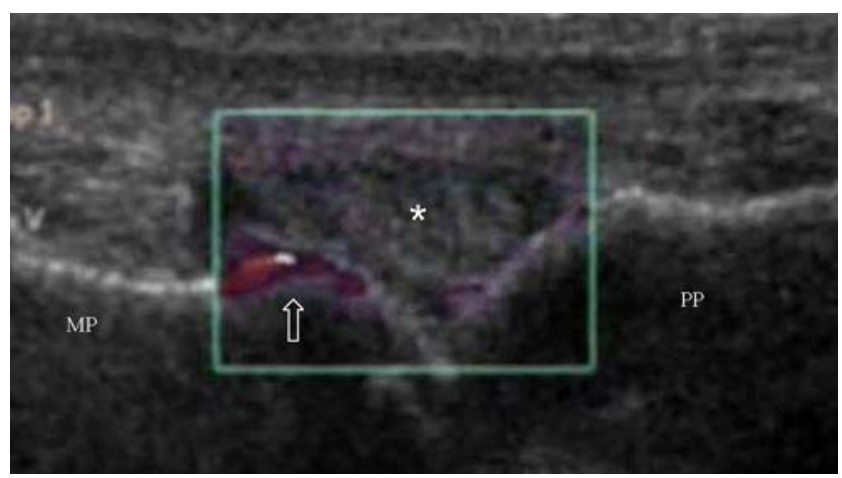

Fig. 14 Static image from video-loop. Apparent Doppler signal from the bone surface of the proximal interphalangeal joint (arrow) in the patient with mild synovial thickening (asterisk). When colour flow settings are set to high sensitivity, flash artefacts can be identified where there are strong reflecting interfaces such as the bone surface. $P P$ proximal phalanx, $M P$ middle phalanx 
same equipment may be incomparable following software or platform upgrades.

Unlike CFDUS, PDUS does not carry specific directional information and will therefore register any flow within the region of interest. Although more recent advances in PDUS mean that some directional information can be imparted in the colour read out, quantification is still based on measuring the amount of colour pixels within the image, rather than an absolute measure of blood flow within the synovial tissue itself. These semi-quantitative measures can be scored either visually or by software analysis. Visual scales are most practicable in the clinical setting. As outlined above, a simple grading system is mild (few scattered vessels), moderate $(<50 \%)$ or severe, $>50 \%$ of the synovial exhibiting increased flow. Various other semi-quantitative scales have been proposed, including that of Klauser et al., who counted the number of visible vessels. Grade 1 is $1-5$ visible signals, grade 2 6-10 and grade 3 more than 11 vessels in the field of view [11]. Computerised methods involve capturing the ultrasound colour image, importing it into a proprietary photo-package and using the software to differentiate and count the number of colour pixels against the greyscale background [16]. The software method requires standardisation of magnification and field of view and is, therefore, troublesome to apply retrospectively.

CFDUS differs from PDUS in that it is dependent on the direction of flow of blood (Fig. 15). The signal acquired carries specific information about the vessel from which it has been obtained in the form of a pressure trace. The precise measurement that is most commonly calculated is the resistive index (RI) (Fig. 16). RI is the difference between systolic and diastolic pressure divided by systolic pressure. Most manufacturers include software within the ultrasound system to calculate the resistive index. A good spectral trace from a vessel within the synovial hypertrophy is acquired. The operator selects one or two pulse cycles from within the trace for analysis. RI is a good marker of

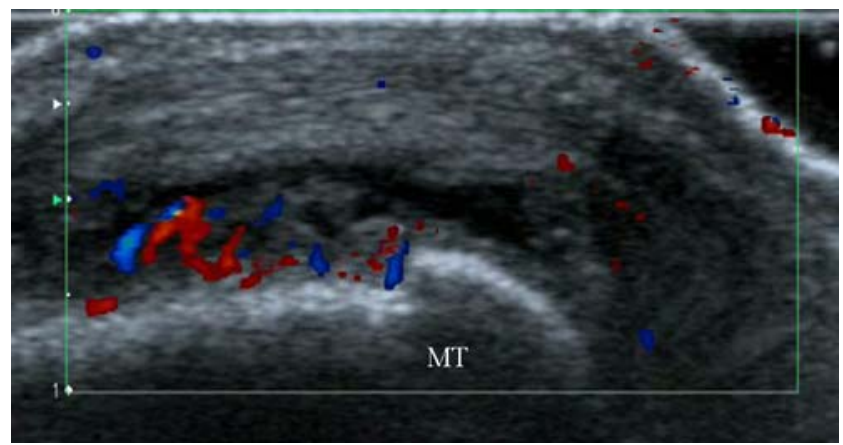

Fig. 15 Colour flow Doppler image from a patient with synovitis of the third metatarso-phalangeal joint. Flow away from the ultrasound probe is depicted in blue and flow towards the probe is red. MT metatarsal high flow resistance: under normal circumstances it is 1 and is reduced by angiogenesis induced by synovitis. In contrast to PDUS, resistive index is a physiological parameter and is, therefore, independent of the equipment used and manufacturer's software. Despite some of the limitations of power Doppler, particularly with regard to serial studies, it is a sensitive measure, which is easy to obtain and broadly reproducible between different operators on the same machine. It appears to correlate reasonably well with contrast-enhanced MRI and clinical findings and allows a rapid assessment, within days, of improvement following treatment.

CFDUS is less sensitive to motion artefact and is a more objective measurement than is PDUS. Problems include difficulties in acquiring a good trace when angiogenesis is sparse and maintaining the fix as measurements are made, particularly if the equipment freezes the image during measurement. The use of beam steering can sometimes help with vessel fixation. CFDUS is insensitive to contrast agents, which diminishes its usefulness in patients with subtle or early synovitis. Changes in RI can also be induced by increasing probe pressure and by changes in temperature. Serial studies should be carried out at controlled temperature. In most cases this can be achieved simply by ensuring the patient has not just come from an abnormally hot or cold environment to room temperature, though some argue that the use of a water bath is the only means of ensuring reliable temperature control. Probe pressure can be controlled in the usual manner by ensuring that there is always a layer of contact gel between probe and skin.

Terslev et al. demonstrated that Doppler ultrasound using changes in resistive index may be used as a quantitative measurement of blood flow and to detect changes following intra-articular steroid injection [17]. The same group also showed that estimates of synovial activity by Doppler ultrasound was comparable with postcontrast MRI, though there was no association between MRI, ultrasound and estimates of inflammation and pain visual analogue scale (VAS) scores. Changes in RI following treatment with anti-tumour necrosis factor (anti-TNF) have also been demonstrated [18]. Varsamidis and co-authors measured resistive index in the wrists of patients with rheumatoid arthritis. The RI improved as the patients went into clinical remission [19]. The authors also suggest that ultrasound may be able to predict relapse, as patients who had a flare up within 6 months had significantly lower RI following treatment than did patients who remained in stable remission.

\section{Ultrasound contrast agents}

Colour Doppler signal from small vessels within the synovium can be augmented by the intravenous adminis- 
Fig. 16 Screen capture during colour Doppler examination of the finger, showing automated calculation of resistive index

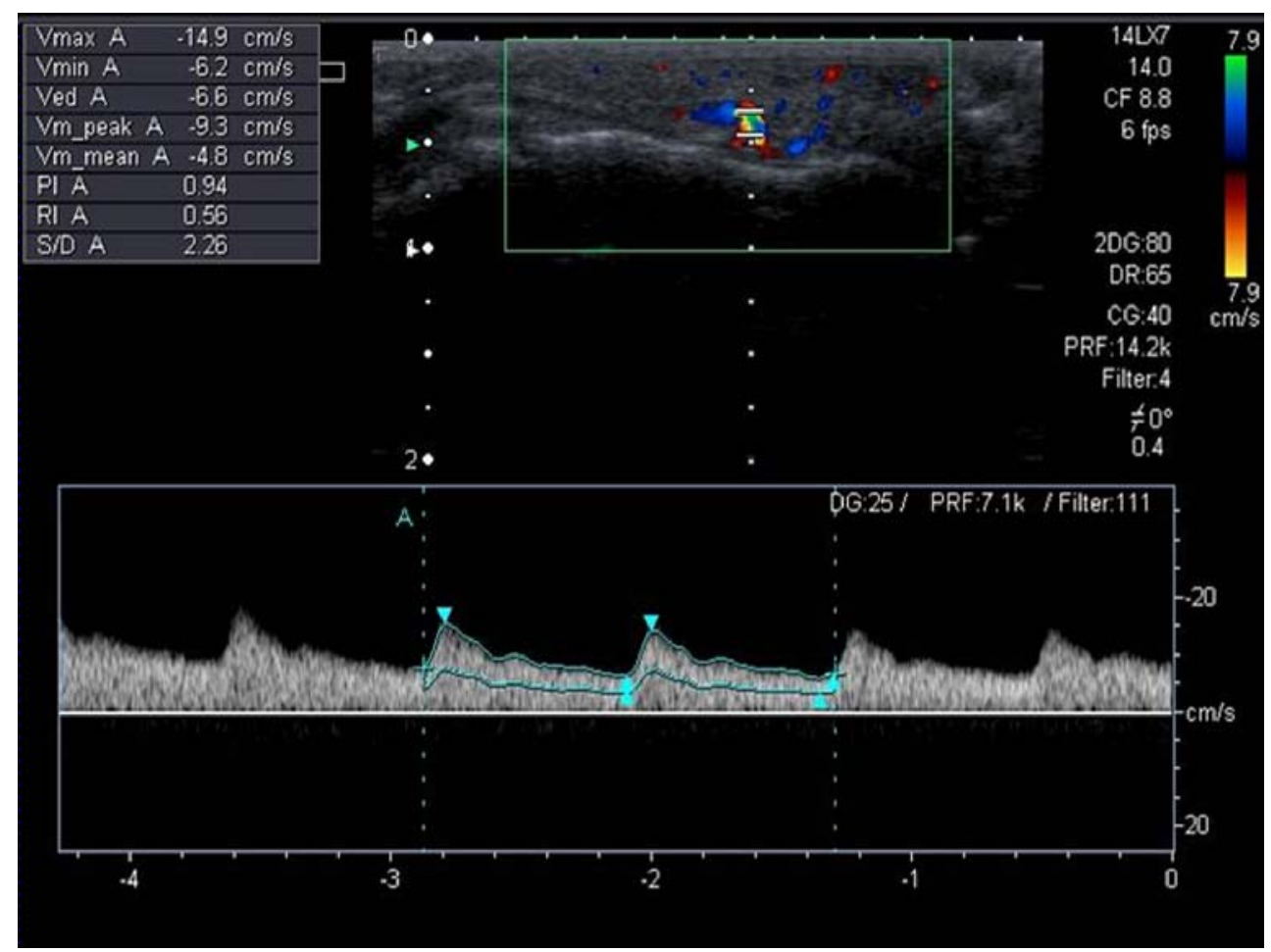

tration of contrast agents. When mixed and injected, these agents release bubbles of gas that are sufficiently small to enter the microcirculation. The earliest compounds were mixtures of galactose and palmitic acid microparticles. When hydrated, galactose releases micro-bubbles of air, which adhere to the irregular surface of the palmitic acid microparticles. The air bubbles are approximately $8 \mu \mathrm{m}$ in diameter and, consequently, are able to enter small capillaries. They remain as a stable level in the circulation for up to $5 \mathrm{~min}$ after injection and increase the Doppler signal by approximately $20 \mathrm{~dB}$ [20]. Second generation US contrast agents use gases other than air, for example sulphur hexafluoride, to provide ultrasonic contrast.

Because ultrasound involves assessment of a relatively small field of view, the short duration of optimal activity of micro-bubbles following a bolus injection means that there will be different concentrations in the synovium of a joint viewed at the beginning of an ultrasound examination compared with one examined at the end. This compares poorly with the single-time snapshot that can be achieved with intravenous administration of MRI contrast agent, where uptake in all of the joints and tendons within the larger field of view can be assessed simultaneously. There are other disadvantages of bolus administration of ultrasound contrast agents, which include a blooming artefact that can occur due to the sudden arrival of a large quantity of micro-bubbles into the ultrasound field and uneven destruction of micro-bubbles by the US wave acting on the large bolus itself. Administering the contrast agents by infusion rather than bolus injection can overcome some of these issues and yield more stable enhancement for up to $20 \mathrm{~min}$. Three-dimensional ultrasound, with maximum intensity projection algorithms, may also help in overcoming some of these disadvantages and provide a better single-time snapshot image of synovial enhancement.

Contrast-enhanced power Doppler ultrasound (CEPDUS) has been used in a number of clinical studies to demonstrate improved detection, over unenhanced ultrasound, of vascularised synovial tissue. Klauser et al. have done much of this work and have classified neovascularisation in patients with rheumatoid arthritis before and after the administration of contrast agent [11]. In the unenhanced study, $65 \%$ of subjects were classified as having normal synovial tissue, grade 0 , and $5 \%$ as severe, grade 4 . Following administration of contrast medium by the infusion technique, only $21 \%$ were deemed to have normal synovial tissue, whilst $22 \%$ increased to grade 4 , with increases in the intermediate groups. Other studies have also demonstrated synovial enhancement and increased detection of synovitis [21] [16]. CEPDUS shows improved correlation over unenhanced power Doppler compared with arthroscopy [22]. Despite this, the therapeutic impact and outcome value of contrast agents requires further study.

\section{Bone erosions}

The identification of bone erosion is a significant step in the natural history of erosive arthritis. The outcome measure- 
ment in rheumatoid arthritis and connective tissue (OMERACT) group has defined bone erosion as an intra-articular discontinuity of the bone surface that is visible in two perpendicular planes. Acute erosions generally have an irregular margin and a poorly defined base, which allows through transmission of sound (Figs. 17 and 18) and are associated with active synovitis. Bone defects that do not have synovitis adjacent to them should be regarded with suspicion, though some may turn out to be true chronic erosions [23]. A common pitfall is the normal depression that is present on the dorsal aspect of the head of the metacarpal. This is smooth, with a clearly defined floor that does not allow through sound transmission and is unassociated with overlying synovitis (Fig. 13).

Erosions in RA are most commonly detected on the radial aspects of the heads of the metacarpals and on the bases of the phalanges. The DIPJs are least involved in RA [23], but more erosions are detected in sero-negative RA and osteoarthritis. In the hands, the second, third and fifth rays are most commonly affected, and, in the feet, the fifth, third and second show the greatest predilection. There are differences between individual joints in the ease by which ultrasound can detect erosions. In the hands, the second and fifth provide the greatest circumferential views at the level of the MCPJs (Fig. 1c). The fourth is the most difficult to examine, especially on its radial and ulnar aspects. Scheel et al. found that ultrasound was superior to MRI in detecting erosions in the proximal interphalangeal joints but was less efficient at the metacarpo-phalangeal joints [24]. Conversely, in Backhaus and colleagues' study of patients with negative plain film findings, erosions dominated in the MCP joints.

Ultrasound appears to be particularly useful in detecting erosions in patients in the early stages of disease [25]. Erosions detected by US and MRI progress to radiographic detection in the majority of cases $[24,26]$ within 1 to

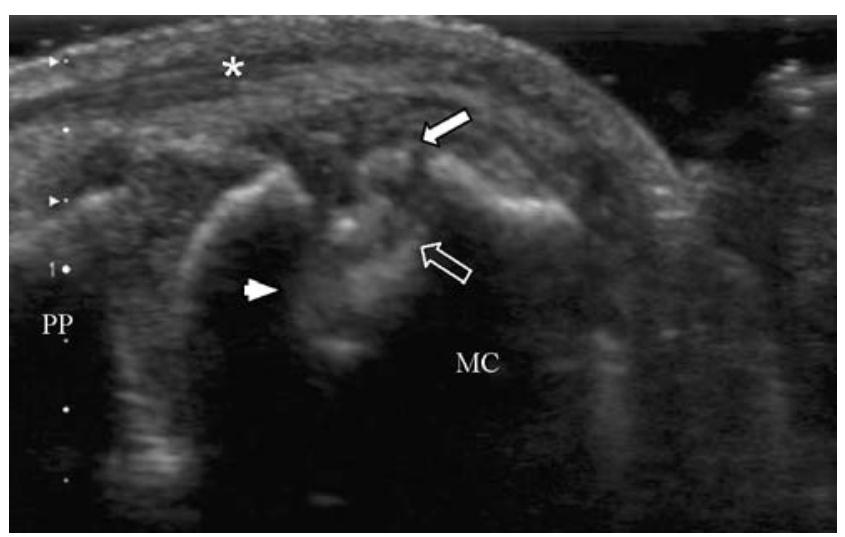

Fig. 17 Sagittal image of a large erosion in the head of a metacarpal. Note the poorly defined floor of the erosion (open arrow) and through sound transmission (arrowhead). Compare with Fig. 13. Note the adjacent synovial thickening (arrow) and the extensor tendon (asterisk). MT metatarsal head, PP proximal phalanx

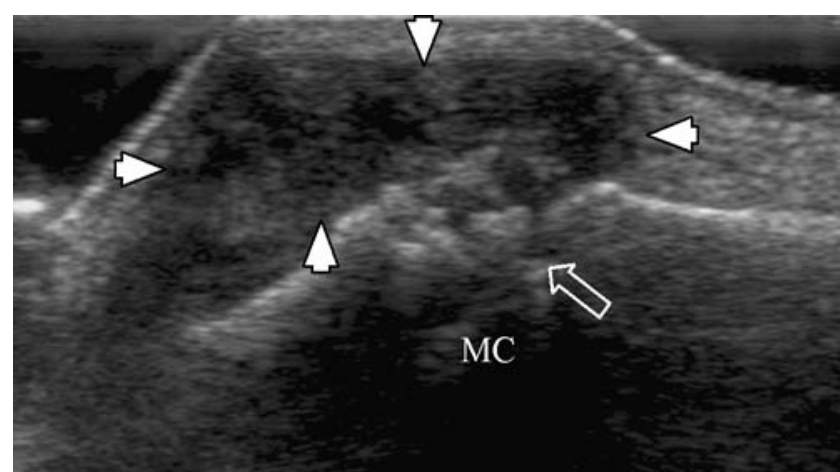

Fig. 18 Moderate synovial thickening (arrowheads) with underlying metacarpal head $(M C)$ erosion (arrow)

2 years. One of the earliest MRI signs of erosion is subchondral bone oedema, and there are, as yet, no studies to show that ultrasound is useful in detecting this. Although the contour of the cortex should be carefully examined for clarity and early defects, ultrasound will not appreciate intraosseous abnormalities that are not associated with an overlying surface defect.

Erosions in gout are larger, also irregular, and lie further away from the joint. Erosions must be distinguished from other causes of peri-articular bone irregularities. Entheseal new bone formation at the site of tendon and ligament insertions occur in seronegative arthritis and can give a similar appearance. They are particularly prevalent at the DIPJs, where erosive changes related to osteoarthritis are also found.

\section{Small joint US following treatment}

Changes in synovial thickening and vascularisation following treatment can occur rapidly. A reduction in the number of vessels identified within the synovium has been shown by US within 3 days of intravenous corticosteroid administration [27]. Direct interarticular corticosteroid administration has also been shown to reduce the number of synovial vessels, which persist for several years, suggesting that they are a real response to therapy and not just a temporary alteration in flow characteristics induced by pressure changes within the joint $[17,28,29]$. Ultrasound has been used to track changes in total synovial volume by greyscale ultrasound imaging [30], power Doppler signal [31-33] and resistive index [31] in response to systemic anti-TNF therapy. Results show a consistent reduction in all these imaging parameters in the early stages of active treatment, though long-term results are less constant. Terslev and colleagues, in a study of wrist and small joint arthritis in patients with rheumatoid disease treated with Etanercept, noted that much of the initial reduction in synovial volume and vascularity and increasing resistive index was lost at 
1 year. Conversely, Fiocco et al., in a study of 27 patients with knee involvement from either rheumatoid or psoriatic arthritis, noted that synovial thickening persisted at 3 months' follow-up but was reduced after 1 year. A reduction in vascularity of the synovium had a more rapid onset and also remained persistently low at 1 year [32].

The limited evidence would seem to suggest that changes in synovial vascularity can be seen very quickly, independent of the type of treatment used. Changes in synovial volume are slower to respond, and measurable differences may take some time to appear in larger joints. In most studies, these changes appear to persist, suggesting that ultrasound can be used as a marker of early improvement. Furthermore, ultrasound has detected improvements in joints felt to be clinical 'non-responders' [30], suggesting an important role for ultrasound in clinical decision making during the early stage of therapy. Most of these studies, however, are unblinded. Evidence that there is a link between early improvement in synovitis detected by US and a reduction in radiographic findings in later years is beginning to emerge [34].

\section{Clinical application of ultrasound in small joint arthritis}

Ultrasound versus clinical examination The role of ultrasound in the detection of sub-clinical synovitis has been examined in a number of studies, most of which demonstrate increased detection rates of intra-articular abnormalities. With MRI as the gold standard, the sensitivity, specificity, and accuracy of US for the detection of synovitis in the MTPJs were $0.87,0.74$, and 0.79 , respectively, and for clinical examination, $0.43,0.89$, and 0.71 , respectively [35]. Wakefield et al. examined the role of ultrasound in detecting sub-clinical synovitis in 1,470 joints in 80 patients with oligoarthritis. Of the clinically unaffected joints, $13 \%$ had synovitis detected by ultrasound; the majority, 79\%, were metatarso-phalangeal joints, and $16 \%$ were metacarpo-phalangeal joints. This meant that one-third of the patients with clinical monoarthritis were upgraded to oligoarthritis $(>1<6$ joints) and a further quarter to polyarthritis ( $>6$ joints) [36]. Szkudlarek and colleagues examined the metatarsophalangeal joints in patients with rheumatoid arthritis and healthy controls. Joint effusion and synovitis were detected by ultrasound in 102 joints compared with positive findings in only eight joints on clinical examination [35]. In both studies non-specific findings, especially effusion, common in the first and second MTPJs, may have skewed those results. The finding of occult synovial disease using ultrasound has not been limited to rheumatoid arthritis but has also been extended to patients with systemic lupus erythematosis and other rheumatological conditions [37].
Ultrasound versus other imaging techniques Ultrasound has been compared with plain radiography and has shown significantly superior synovitis detection rates. This is unsurprising, as signs of synovitis on plain radiography, including peri-articular osteoporosis, joint space widening and soft tissue swelling, may be difficult to detect and are frequently overlooked. Backhaus et al. demonstrated a fourfold superiority with ultrasound, compared to the plain radiographic findings of peri-articular osteoporosis and soft tissue swelling [23]. In that study, patients with a variety of rheumatological disorders, who had negative findings on plain radiography, had synovitis demonstrated by ultrasound in all cases, whereas MRI was positive in $84 \%$. In the same group, ultrasound detected erosions in 15 patients compared to 26 detected by MRI. More were found in the proximal interphalangeal joints than by MRI, whereas the reverse was true for metacarpo-phalangeal joints, though other authors have not confirmed this [38].

The use of low-field MRI, sequence choice and the use of lower resolution dynamic rather than static post-contrast MR images may have contributed to the apparent superiority of ultrasound in detecting synovitis. Furthermore, both joint effusion and synovial thickening were interpreted as 'synovitis', which might have also increased detection rates for ultrasound over MRI [23]. Conversely, the analogue ultrasound equipment used would be regarded as relatively low specification by modern standards. Indeed, in a followup to that study, published 7 years after the original, ultrasound detected more erosions than MRI did, in $49 \%$ of patients ( $9 \%$ at baseline) compared to $32 \%$ detected by MRI (27\% at baseline) [24]. In the follow-up study the original low-resolution analogue ultrasound equipment was replaced by a more advanced digital system. Although there was also an increase in the number of erosions detected by conventional radiography, from 5 to 33 joints, it is more likely that technological improvements in the ultrasound equipment accounted for the greater detection of erosions and that many had been missed at the baseline study. It is, therefore, imperative that superior resolution equipment is used for rheumatological examination. Other studies have confirmed the superiority of ultrasound over radiography in the detection of erosions [39], independent of the number of projections used [25].

Contrast-enhanced ultrasound and MRI have been compared in a small number of studies. Terslev et al. compared Doppler ultrasound with quantification of both the colour fraction and RI with synovial thickness on postcontrast MRI [8]. Dynamic enhanced MRI detected more inflamed MCP joints than ultrasound did, but the reverse was true for the wrist. Szkudlarek and colleagues compared contrast-enhanced ultrasound using the bolus technique with post-contrast MRI in actively inflamed MCPJs [40]. The MRI enhancement rates at $55 \mathrm{~s}$ after injection were 


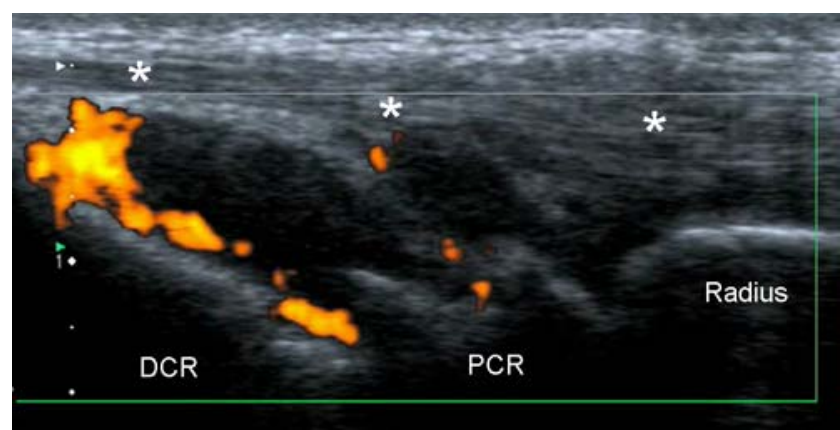

Fig. 19 Long axis-view on the dorsal aspect of the wrist deep to the extensor tendon (asterisks). Intense Doppler signal intensity is detected in the distal portion of the joint. DCR distal carpal row, $P C R$ proximal carpal row

used, as the authors found this to correlate most closely with histological features of synovial inflammation [4145]. They concluded that there was good correlation between PDUS and MRI, but noted that the diagnostic impact of contrast enhancement was limited to a relatively small number of patients.

Although these studies have attempted to compare ultrasound and MRI contrast enhancement directly, it should be appreciated that there is an essential difference between enhancing synovium on MRI and measurable angioneogenesis on Doppler ultrasound. Enhanced MRI identifies contrast agent in the intravascular, extracellular and joint spaces, the latter depending on the time allowed for diffusion. CEPDUS recognises only new vessel formation within inflamed synovium. It is well recognized that synovial thickening (presumably detectable on enhanced MRI) can be present on ultrasound examination without associated increased vascularity. While it is, therefore, valid to draw some parallels between the two imaging tech niques, direct comparison misses vital information on the physiology of the synovium, and differences between them should not be regarded as false negatives or false positives.

Klarlund and co-workers compared MRI, plain radiographs and scintigraphy in 55 patients, followed-up for 1 year. MRI detected progression of erosions more often than radiography did, but ultrasound was not used in that

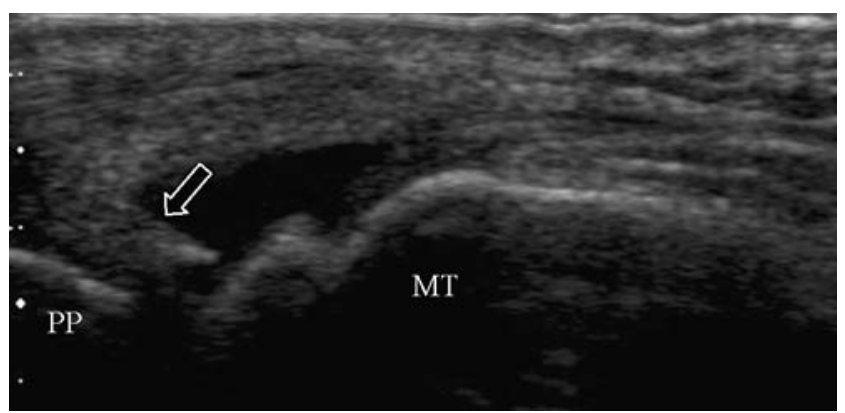

Fig. 20 Needle introduced into the second metatarso-phalangeal joint (arrow) prior to aspiration and corticosteroid injection. MT metatarsal head, $P P$ proximal phalanx

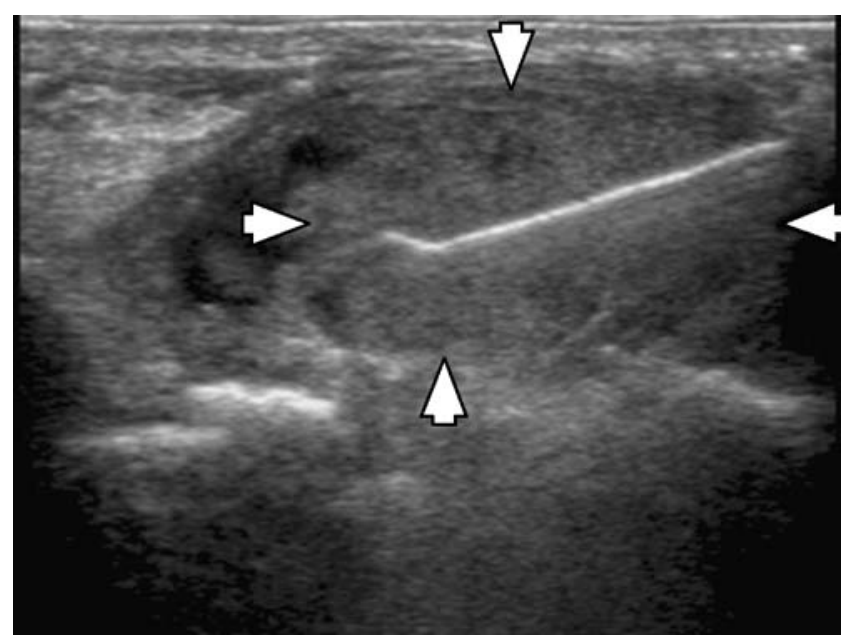

Fig. 21 A synovial mass (arrowheads) undergoing biopsy. Ultrasound allows accurate placement of a small biopsy needle within the synovial tissue

study [46]. Low- and high-field MRI have also been directly compared with plain radiography [47]. The different field strengths had equivalent erosion detection rates, and both were superior to radiography.

\section{Advantages and disadvantages of ultrasound}

The current literature suggests that ultrasound is superior to MRI at detecting small quantities of fluid within the joint. There may be improved detection of erosions at the proximal interphalangeal joints and in the wrist and, although ultrasound struggles with some areas of MCP joints, particularly the radial and ulnar aspects of the fourth finger, overall erosion detection by US at the MCPJs is comparable to that by MRI [38]. US demonstrates angioneogenesis in active synovitis directly (Fig. 19), whereas MRI requires injection of contrast medium. US is a dynamic technique; the examination can be tailored to include further involved joints following an up-to-date history with the patient present There are fewer problems with asymptomatic abnormalities, as these can be clinically correlated during the examination. Unlike MR, US is readily suited to guiding intervention, such as small joint injection or synovial biopsy (Figs. 20, 21). There is also increasing evidence that patients prefer ultrasound to MRI [48]. This may be related to claustrophobia but equally to the human interaction that occurs during an ultrasound examination.

Conversely, ultrasound has difficulty with large deep joints, superficial joints when there are significant deformities, and in the assessment of articular cartilage. US is a relatively time-consuming procedure, especially if multiple joints are examined. The images acquired can be re-read and reclassified by other readers at a later date, but there is 
no ability to identify abnormalities overlooked and not imaged at the time of the original examination. MR images can be obtained remotely, reviewed, re-read and scored at a later date and preserved for serial comparison. Increasing use of ultrasound video-loops and 3-D ultrasound may assist in this regard in the future. MRI demonstrates singletime shot synovial enhancement in the entire field of view, which can include all the important small joints of the hands and wrists as well as adjacent tendons and ligaments. Although the time taken to review MRI images of the hand and wrist for diagnostic purposes is probably shorter than the time taken to complete an ultrasound examination, a full MRI score such as the rheumatoid arthritis MRI scoring system (RAMRIS), which is a more close approximation to ultrasound, takes considerably longer. Despite this, there is a strong argument that, for research studies of different therapeutic regimens in patients with rheumatoid arthritis, plain films and MRI provide more robust serial assessment and will remain the gold standard for some time.

\section{Conclusions}

In routine rheumatological practice, ultrasound of the small joints of the hands and feet accurately detects occult synovial disease and allows classification of the extent and activity of synovitis as well as superior detection of erosions in comparison with radiography. US can guide intra-articular therapy as well as assess its response and the response to systemic treatment. Future goals for ultrasound trials need to include further validation studies, studies of diagnostic and therapeutic impact and longer term outcomes from clinical and therapeutic decisions based on the ultrasound examinations.

\section{References}

1. Scheel AK, Hermann KG, Kahler E, et al. A novel ultrasonographic synovitis scoring system suitable for analyzing finger joint inflammation in rheumatoid arthritis. Arthritis Rheum 2005; 52 : 733-743.

2. Ostergaard M, Szkudlarek M. Ultrasonography: a valid method for assessing rheumatoid arthritis? Arthritis Rheum 2005; 52: 681-686.

3. Tan AL, Tanner SF, Conaghan PG, et al. Role of metacarpophalangeal joint anatomic factors in the distribution of synovitis and bone erosion in early rheumatoid arthritis. Arthritis Rheum 2003; 48: 1214-1222.

4. Hau M, Schultz H, Tony H-P, et al. Evaluation of pannus and vascularization of the metacarpophalangeal and proximal interphalangeal joints in rheumatoid arthritis by high-resolution ultrasound (multidimensional linear array). Arthritis Rheum 1999; 42:2303-2308.
5. Bade H, Koebke J, z. Nieden A. Radiologic anatomy of the metacarpophalangeal joints II to V. Surg Radiol Anat 1998; V19: 327.

6. Naredo E, Moller I, Moragues C, et al. Interobserver reliability in musculoskeletal ultrasonography: results from a "Teach the Teachers" rheumatologist course. Ann Rheum Dis 2006; 65: 14 19.

7. Schmidt WA, Schmidt H, Schicke B, Gromnica-Ihle E. Standard reference values for musculoskeletal ultrasonography. Ann Rheum Dis 2004; 63: 988-994.

8. Terslev L, Torp-Pedersen S, Savnik A, et al. Doppler ultrasound and magnetic resonance imaging of synovial inflammation of the hand in rheumatoid arthritis: a comparative study. Arthritis Rheum 2003; 48: 2434-2441.

9. Bresnihan B. Rheumatoid arthritis: principles of early treatment. J Rheumatol Suppl 2002; 66: 9-12.

10. Szkudlarek M, Court-Payen M, Jacobsen S, Klarlund M, Thomsen HS, Ostergaard M. Interobserver agreement in ultrasonography of the finger and toe joints in rheumatoid arthritis. Arthritis Rheum 2003; 48: 955-962.

11. Klauser A, Frauscher F, Schirmer M, et al. The value of contrastenhanced color Doppler ultrasound in the detection of vascularization of finger joints in patients with rheumatoid arthritis. Arthritis Rheum 2002; 46: 647-653.

12. Terslev L, Torp-Pedersen S, Bang N, Koenig MJ, Nielsen MB, Bliddal H. Doppler ultrasound findings in healthy wrists and finger joints before and after use of two different contrast agents. Ann Rheum Dis 2005; 64: 824-827.

13. Walther M, Harms H, Krenn V, Radke S, Faehndrich TP, Gohlke F. Correlation of power Doppler sonography with vascularity of the synovial tissue of the knee joint in patients with osteoarthritis and rheumatoid arthritis. Arthritis Rheum 2001; 44: 331-338.

14. Walther M, Harms H, Krenn V, Radke S, Kirschner S, Gohlke F. Synovial tissue of the hip at power Doppler US: correlation between vascularity and power Doppler US signal. Radiology 2002; 225: 225-231.

15. Koski JM, Saarakkala S, Helle M, Hakulinen U, Heikkinen JO, Hermunen H. Power Doppler ultrasonography and synovitis. Correlating ultrasound imaging with histopathological findings and evaluating the performance of ultrasound equipments. Ann Rheum Dis 2006; 65: 1590-1595.

16. Qvistgaard E, Rogind H, Torp-Pedersen S, Terslev L, DanneskioldSamsoe B, Bliddal H. Quantitative ultrasonography in rheumatoid arthritis: evaluation of inflammation by Doppler technique. Ann Rheum Dis 2001; 60: 690-693.

17. Terslev L, Torp-Pedersen S, Qvistgaard E, Danneskiold-Samsoe B, Bliddal H. Estimation of inflammation by Doppler ultrasound: quantitative changes after intra-articular treatment in rheumatoid arthritis. Ann Rheum Dis 2003; 62: 1049-1053.

18. Hau M, Kneitz C, Tony H-P, Keberle M, Jahns R, Jenett M. High resolution ultrasound detects a decrease in pannus vascularisation of small finger joints in patients with rheumatoid arthritis receiving treatment with soluble tumour necrosis factor alpha receptor (etanercept). Ann Rheum Dis 2002; 61:55-58.

19. Varsamidis K, Varsamidou E, Tjetjis V, Mavropoulos G. Doppler sonography in assessing disease activity in rheumatoid arthritis. Ultrasound Med Biol 2005; 31: 739-743.

20. Calliada F, Campani R, Bottinelli O, Bozzini A, Sommaruga MG. Ultrasound contrast agents: basic principles. Eur J Radiol 1998; 27 [Suppl 2]: S157-S160.

21. Magarelli N, Guglielmi G, Di Matteo L, Tartaro A, Mattei PA, Bonomo L. Diagnostic utility of an echo-contrast agent in patients with synovitis using power Doppler ultrasound: a preliminary study with comparison to contrast-enhanced MRI. Eur Radiol 2001; 11: 1039-1046. 
22. Fiocco U, Ferro F, Cozzi L, et al. Contrast medium in power Doppler ultrasound for assessment of synovial vascularity: Comparison with arthroscopy. J Rheumatol 2003; 30: 2176.

23. Backhaus M, Kamradt T, Sandrock D, et al. Arthritis of the finger joints: a comprehensive approach comparing conventional radiography, scintigraphy, ultrasound, and contrast-enhanced magnetic resonance imaging. Arthritis Rheum 1999; 42: 1232-1245.

24. Scheel AK, Hermann KGA, Ohrndorf S, et al. Prospective 7 year follow up imaging study comparing radiography, ultrasonography, and magnetic resonance imaging in rheumatoid arthritis finger joints. Ann Rheum Dis 2006; 65: 595-600.

25. Lopez-Ben R, Bernreuter WK, Moreland LW, Alarcon GS. Ultrasound detection of bone erosions in rheumatoid arthritis: a comparison to routine radiographs of the hands and feet. Skeletal Radiol 2004; 33: 80-84.

26. Ostergaard M, Hansen M, Stoltenberg M, et al. New radiographic bone erosions in the wrists of patients with rheumatoid arthritis are detectable with magnetic resonance imaging a median of two years earlier. Arthritis Rheum 2003; 48: 2128-2131.

27. Teh J, Stevens K, Williamson L, Leung J, McNally EG. Power Doppler ultrasound of rheumatoid synovitis: quantification of therapeutic response. Br J Radiol 2003; 76: 875-879.

28. Filippucci E, Farina A, Carotti M, Salaffi F, Grassi W. Grey scale and power Doppler sonographic changes induced by intraarticular steroid injection treatment. Ann Rheum Dis 2004; 63: 740-743.

29. Newman JS, Laing TJ, McCarthy CJ, Adler RS. Power Doppler sonography of synovitis: assessment of therapeutic responsepreliminary observations. Radiology 1996; 198: 582-584.

30. Ribbens C, Andre B, Marcelis S, et al. Rheumatoid hand joint synovitis: gray-scale and power Doppler US quantifications following anti-tumor necrosis factor-\{alpha\} treatment: pilot study. Radiology 2003; 229: 562-569.

31. Terslev L, Torp-Pedersen S, Qvistgaard E, et al. Effects of treatment with etanercept (Enbrel, TNRF:Fc) on rheumatoid arthritis evaluated by Doppler ultrasonography. Ann Rheum Dis 2003; 62: 178-181.

32. Fiocco U, Ferro F, Vezzu M, et al. Rheumatoid and psoriatic knee synovitis: clinical, grey scale, and power Doppler ultrasound assessment of the response to etanercept. Ann Rheum Dis 2005; 64: 899-905.

33. Filippucci E, Iagnocco A, Salaffi F, Cerioni A, Valesini G, Grassi W. Power Doppler sonography monitoring of synovial perfusion at the wrist joints in patients with rheumatoid arthritis treated with adalimumab. Ann Rheum Dis 2006; 65: 1433-1437.

34. Taylor PC. Serum vascular markers and vascular imaging in assessment of rheumatoid arthritis disease activity and response to therapy. Rheumatology 2005; 44: 721-728.

35. Szkudlarek M, Narvestad E, Klarlund M, Court-Payen M, Thomsen HS, Ostergaard M. Ultrasonography of the metatarsophalangeal joints in rheumatoid arthritis: comparison with magnetic resonance imaging, conventional radiography, and clinical examination. Arthritis Rheum 2004; 50: 2103-2112.

36. Wakefield RJ, Green MJ, Marzo-Ortega H, et al. Should oligoarthritis be reclassified? Ultrasound reveals a high prevalence of subclinical disease. Ann Rheum Dis 2004; 63: 382-385.

37. Iagnocco A, Ossandon A, Coari G, et al. Wrist joint involvement in systemic lupus erythematosus. An ultrasonographic study. Clin Exp Rheumatol 2004; 22: 621-624.

38. Ostergaard M, Ejbjerg B, Szkudlarek M. Imaging in early rheumatoid arthritis: roles of magnetic resonance imaging, ultrasonography, conventional radiography and computed tomography. Best Pract Res Clin Rheumatol 2005; 19: 116.

39. Wakefield RJ, Gibbon WW, Conaghan PG, et al. The value of sonography in the detection of bone erosions in patients with rheumatoid arthritis: a comparison with conventional radiography. Arthritis Rheum 2000; 43: 2762-2770.

40. Szkudlarek M, Court-Payen M, Strandberg C, Klarlund M, Klausen T, Ostergaard M. Contrast-enhanced power Doppler ultrasonography of the metacarpophalangeal joints in rheumatoid arthritis. Eur Radiol 2003; 13: 163-168.

41. Ostergaard M, Stoltenberg M, Løvgreen-Nielsen P, Volck B, Sonne-Holm S, Lorenzen I. Quantification of synovistis by MRI: correlation between dynamic and static gadolinium-enhanced magnetic resonance imaging and microscopic and macroscopic signs of synovial inflammation. Magn Reson Imaging 1998; 16: 743-754.

42. Tamai K, Yamato M, Yamaguchi T, Ohno W. Dynamic magnetic resonance imaging for the evaluation of synovitis in patients with rheumatoid arthritis. Arthritis Rheum 1994; 37: 1151-1157.

43. Gaffney K, Cookson J, Blades S, Coumbe A, Blake D. Quantitative assessment of the rheumatoid synovial microvascular bed by gadolinium-DTPA enhanced magnetic resonance imaging. Ann Rheum Dis 1998; 57: 152-157.

44. König H, Sieper J, Wolf KJ. Rheumatoid arthritis: evaluation of hypervascular and fibrous pannus with dynamic MR imaging enhanced with Gd-DTPA. Radiology 1990; 176: 473-477.

45. Klarlund M, Ostergaard M, Rostrup E, Skjodt H, Lorenzen I. Dynamic magnetic resonance imaging of the metacarpophalangeal joints in rheumatoid arthritis, early unclassified polyarthritis, and healthy controls. Scand J Rheumatol 2000; 29: 108-115.

46. Klarlund M, Ostergaard M, Jensen KE, et al. Magnetic resonance imaging, radiography, and scintigraphy of the finger joints: one year follow up of patients with early arthritis. Ann Rheum Dis 2000; 59: 521-528.

47. Taouli B, Zaim S, Peterfy CG, et al. Rheumatoid arthritis of the hand and wrist: comparison of three imaging techniques. AJR Am J Roentgenol 2004; 182: 937-943.

48. Middleton WD, Payne WT, Teefey SA, Hildebolt CF, Rubin DA, Yamaguchi K. Sonography and MRI of the shoulder: comparison of patient satisfaction. AJR Am J Roentgenol 2004; 183: 14491452. 